\title{
Fitness: Philosophical Problems
}

\author{
A3443 \\ Ramsey, Grant \\ Grant Ramsey \\ Department of Philosophy, University of Notre Dame \\ Notre Dame, IN \\ USA \\ Pence, Charles H. \\ Charles H. Pence \\ Program in History and Philosophy of Science, University of Notre Dame \\ Notre Dame, IN \\ USA
}




\title{
Fitness: Philosophical Problems
}

\author{
Abstract: [120-150 words] \\ Fitness plays many roles throughout evolutionary theory, from a measure of populations \\ in the wild to a central element in abstract theoretical presentations of natural selection. \\ It has thus been the subject of an extensive philosophical literature, which has primarily \\ centered on the way to understand the relationship between fitness values and \\ reproductive outcomes. If fitness is a probabilistic or statistical quantity, how is it to be \\ defined in general theoretical contexts? How can it be measured? Can a single \\ conceptual model for fitness be offered that applies in all biological cases, or must fitness \\ measures be case-specific? Philosophers have explored these questions over the last \\ several decades, largely in the context of an influential definition of fitness proposed in \\ the late 1970s: the propensity interpretation. This interpretation as first described \\ undeniably suffers from significant difficulties, and debate regarding the tenability of \\ amendments and alternatives to it remains unsettled.
}

Keywords: environment, evolution, fitness, drift, measurement, natural selection, propensity interpretation, tautology

\section{Key Concepts:}

- Actual offspring production is an unsuitable definition of fitness.

- The propensity interpretation of fitness was proposed as a way to base fitness on expected, rather than actual, offspring production. 
- The propensity interpretation is problematic, as it makes fitness difficult to measure, relies centrally on the arithmetic mean, and fails to take into account local environmental factors.

- Some philosophers have rejected the propensity interpretation in favor of an ecological fitness concept or a purely statistical fitness concept.

- Others have attempted to modify the propensity interpretation in order to resolve its problems.

- The debate over which of these concepts of fitness best solves the philosophical problems at hand remains unsettled.

\section{Introduction}

Few concepts have elicited such a long and heated debate in the philosophy of biology as that of fitness. Although fitness was not a central theoretical term in Darwin's (1859) original articulation of this theory of evolution by natural, it quickly rose to importance. Evolution by natural selection is now standardly presented (e.g., Lewontin 1970) as requiring three conditions: variation, heritability, and fitness differences. Empirically, evolutionary studies are thus to a large degree studies of fitness differences and their consequences. And theoretically, fitness, symbolized as $W$, is a key variable in the mathematical formulations of evolutionary theory. See also: DOI:

10.1038/npg.els.0001745

The fact that biologists model and measure fitness seems to imply that fitness is a (measurable) property and that some biological entities are bearers of this property. But this leads to several questions. What sort of property is fitness? Is it, say, a causal 
property, allowing one to justifiably say that some biological entities of a particular kind outcompeted the others because (where this because is understood causally) they were fitter? And what sort of biological entities can legitimately be considered bearers of the property of fitness? Can genes, organisms, populations, or even species have fitness values? The last of these questions is strongly linked with the "levels/units of selection problem" and we will thus not focus on it here. In particular, the question of whether to define the fitness as a property of token organisms or of types of organisms has been the subject of some debate. Our focus will instead be on the nature of the property of individual fitness. Similarly, we will not deal with issues of individuation. If one is to compare the fitness of two individuals, then one must have a way of identifying them as distinct individuals. Although individuality is fairly obvious for some taxa (e.g., alligators or apple trees) it is far from obvious in others (e.g., aphids or aspens). We will simply take for granted the individualization of organisms in what follows, but we do want to emphasize that we recognize it as an important issue related to that of fitness. See Chapter 4 of Godfrey-Smith (2009) for an excellent introduction to issues in reproduction and individuality. See also: DOI: 10.1038/npg.els.0001712, DOI:

10.1038/npg.els.0003463, DOI: 10.1038/npg.els.0005447

Setting aside the arguments over individuation and the levels of selection and assuming that organisms are one of the kinds of entities that can bear fitness, we can begin to ask what sort of property organismic fitness might be. Fitness is often broken down into (at least) two components, viability and fecundity. The former concerns survival to maturity, while the latter concerns the production of offspring once mature. Throughout the history of evolutionary theory fitness has occasionally been considered to 
be the organism's total offspring production, the combination of these factors. The fitness of any organism, on this view, is just the number of offspring that it actually has - and, if it is reproductively successful, its fitness will "ratchet up" with each offspring it bears.

This actual reproductive success understanding of fitness (henceforth "realized fitness") has the merits of being simple and readily measurable. But it has some significant problems. Fitness is often used to explain evolutionary outcomes - organisms of one type are said to out-reproduce those of another type because the former are fitter than latter. But if fitness just is realized fitness, then the claim that "the fitter organisms out-reproduce the less-fit" is equivalent to "the organisms that reproduce more outreproduce those which reproduce less," a simple tautology. The argument is an old one in evolutionary theory (see, e.g., Butler 1879, pp. 351-355), was taken up occasionally by philosophers (e.g., Popper 1974), and is still utilized by creationists (Pennock 1999, p. 101). Understanding fitness as realized fitness, then, results in an arguably fatal flaw. In addition to the tautology problem, another problem with equating fitness with actual reproductive success is that it precludes distinguishing evolutionary responses due to fitness differences from genetic drift. It is generally understood that populations exhibit drift, and that the magnitude of drift increases as the population size decreases. But if fitness is equated with evolutionary outcomes, it can no longer be contrasted with drift, either as a distinct kind of outcome or as a distinct kind of cause. It is for these reasons that philosophers have sought conceptions of fitness that are not equated with evolutionary outcomes. The philosophical debate has centered on a position known as the propensity interpretation of fitness, which will be the focus of what follows. 


\section{The Propensity Interpretation of Fitness}

The propensity interpretation of fitness (PIF) was introduced in the late 1970s by Brandon (1978) and Mills and Beatty (1979). They argued that instead of considering the fitness of organisms to be identified with the actual number of offspring produced, fitness should instead be equated with the probabilistic propensity to produce offspring - a distribution of probability values describing how likely it is that an organism will produce no offspring, one offspring, etc. The PIF, then, appears to solve the problems just discussed. First, if fitness is a propensity, then it is a kind of dispositional property, similar to other dispositional properties like solubility. The fact that an object is solublea grain of salt, say - does not mean that it actually will dissolve, but that it would, were it placed into the appropriate circumstances. Similarly, the fitness of an organism does not determine a particular reproductive outcome (unless one particular value in the distribution has a probability of 1), but describes how that organism is disposed to reproduce. And to the degree to which outcomes can be explained by probabilistic causes (itself a contentious philosophical question; see also Mayr 1961), the organism's actual reproductive success is causally explained by its fitness.

If fitness is a dispositional property then, unlike in the case of realized fitness, drift can be distinguished from selection. There are debates about how, precisely, drift is to be distinguished from selection. Some, for example, argue that selection and drift represent distinct causes (Hodge 1987; Millstein 2006), while others hold that the distinction is best understood in terms of outcomes (Matthen and Ariew 2002; Walsh 2007). But regardless of how one attempts to make this distinction, it is clear that if 
fitness is understood in terms of realized outcomes only, then the theoretical resources for distinguishing selection and drift do not exist.

As an example of how drift can be distinguished from selection, consider that the PIF provides a probability distribution over possible values of offspring production. An organism may have a probability of 0.2 of having 0 offspring, a probability of 0.1 of having 1 offspring, etc. Now consider how we would describe the reproductive output of all the individuals with a particular trait (having brown, as opposed to grey fur). This will again be a probability distribution, derivable from the individual-level distributions. If we take the arithmetic mean of the distribution, we are provided with the expected number of copies of the trait that will appear in the next generation. Assuming the trait is perfectly heritable, and that there is no migration or mutation (and other usual caveats), fitness values thus provide us with expectation values for the proportion of individuals possessing the traits found in the next generation (the ratio of individuals with brown fur, say). Depending on how drift is understood (a topic too far afield for us here), deviations from those expectation values either count as genetic drift or provide evidence for drift (though the nature and weight of this evidence will vary depending on the precise definition of genetic drift in use). And, because there will be fewer individuals to be sampled from in small populations, there will be a larger deviation from this expectation value in these populations. (For the same reason, the deviation from the expectation value of a fair coin will tend to decrease with the number of flips. You would predict a large deviation from 50-50 for a sequence of three flips, but a small deviation for 300 flips.) The PIF thus both allows for the conceptual distinction between drift and selection, and also reflects the observation that drift tends to have a higher magnitude in small 
populations. See also: DOI: 10.1038/npg.els.0001698, DOI:

10.1002/9780470015902.a0001772.pub3

\section{Challenges to the Propensity Interpretation of Fitness}

Despite the clear benefits of the PIF over realized fitness, the PIF as originally described is not without challenges. Let's consider here some of the key challenges to the PIF. We will then discuss responses to these challenges in the next two sections.

Challenge 1: The PIF makes fitness unknowable. In practice, the fitness of individual organisms will be difficult to ascertain under the PIF framework, and fitness values cannot be directly determined by observing a small number of cases of actual offspring production. Given that the propensity proposed by the PIF manifests as a probability distribution over all possible numbers of offspring, a large number of similar

(if not clonal) organisms in similar environments will need to be observed in order for us to have any confidence in our estimate of an individual's fitness. In almost all biological cases, this will be exceedingly difficult.

\section{Challenge 2: The arithmetic mean is not always a good way of modeling the} propensity. While describing the probability distribution associated with the reproductive success of an organism is useful, there are also many circumstances in which fitness must be considered as a single numerical value, to enable comparisons between the fitnesses of different organisms. As mentioned above, the traditional way to formalize this in the PIF is to let the numerical value of fitness be equal to the arithmetic mean or expectation value of the probability distribution. It has long been known, however, that the arithmetic mean is not always the best predictor of future population success (see, e.g., Lewontin 
and Cohen 1969, Gillespie 1974), and this problem was even noted by some of the creators of the PIF (Beatty and Finsen 1989). In some of these cases, a geometric mean (or some other measure) may offer a better prediction of a trait's future frequency. The defender of the PIF must, therefore, either provide a method for reducing this probability distribution to a single numerical value - one that takes into account the fact that the arithmetic mean will not always be the best choice - or they must argue why such a reduction is not necessary for the cogency of the PIF.

\section{Challenge 3: How the PIF is understood changes with differing environmental} circumstances, population structure, etc. Many authors (Rosenberg 1982; Sober 2001; Matthen and Ariew 2002; Ariew and Lewontin 2004; Bouchard and Rosenberg 2004) have argued that one basic theoretical premise in the PIF is flawed. The PIF assumes, these authors argue, that a single definition of the concept of fitness can be adequate to describe the fitness of individual organisms in all biological circumstances, and this is a false assumption. Some have argued further that fitness can only be specified by relativizing to a particular set of fitness components of interest in a particular set of local environmental circumstances, or even to a particular pair of organisms of interest. As environment, population structure, and other local factors change, the very definition of fitness, they argue, must change with them. In order to salvage the PIF, then, its defenders must show that it is sensitive in the appropriate way to all these influences, and that the theoretical worries raised by these critics concerning the possibility of a general measure of fitness are unfounded.

Challenge 4: What facts determine the probability distribution in the PIF? What are its environmental scope and time-frame? In the initial description of the PIF above, 
the precise facts that are to be used to fix the values of the probability distribution were left unspecified. Are these only facts about currently living organisms and their projected offspring numbers? In order to deal with mutations with effects on future generations (Crow and Kimura 1956, Ahmed and Hodgkin 2000), should the PIF take into account descendants in later generations? Building on the last challenge, which environmental factors should be considered part of fitness calculations, and which should be considered "external" to individual fitness? The defender of the PIF will need to provide general answers to these questions in order for the probability distribution to be well founded in all biological cases.

\section{Responses Abandoning the PIF}

One obvious way to respond to this set of rather thorny challenges is by abandoning the PIF entirely, and developing a new way to understand individual fitness. One such attempt was put forth by Bouchard and Rosenberg (2004). They argue that the focus on propensities - in particular, the focus on measuring fitness using propensities - is mistaken. The fundamental notion of fitness is, rather, comparative. The best we can hope for in a general interpretation of fitness, then, defines fitness in terms of two organisms a and $\mathrm{b}$, and an environment $\mathrm{E}$ :

$\mathrm{a}$ is fitter than $\mathrm{b}$ in $\mathrm{E}=\mathrm{a}$ 's traits result in its solving the design problems set by E more fully than b's traits. (Bouchard and Rosenberg 2004, p. 699)

They call this notion "ecological fitness," and endeavor to respond to several of the difficult philosophical issues inherent both in providing a definition of "design problems" and measuring how well organisms might "solve" those problems. See also: DOI: 10.1038/npg.els.0004166 
Another highly influential attempt to develop an alternative to the PIF has come to be known as the "statisticalist" interpretation of evolutionary theory. As framed in Matthen and Ariew (2002; see also Walsh, Lewens, and Ariew 2002; Pigliucci and Kaplan 2006, ch. 1), the PIF's troubles come from a conflation of two senses of "fitness" that ought, in fact, to be kept distinct. The "vernacular" notion of fitness is a highly general, usually descriptive concept of fitness. It appears in some characterizations of natural selection such as the "principle of natural selection" (i.e., that "if A has higher fitness than B in E, then A will probably outcompete B over time," see Bouchard and Rosenberg 2004). This is to be contrasted with the "predictive" notion of fitness as it appears in population genetics or mathematical biology. Predictive fitness is strictly specified, only valid in very precise circumstances, and quantifiable. See also: DOI: 10.1002/9780470015902.a0001737.pub2

The trouble with the propensity interpretation, Matthen and Ariew argue, is that it mistakenly believes these two notions of fitness to be connected - that is, both that the propensity itself is a characterization of vernacular fitness, and that the expected number of offspring given that propensity is a characterization of predictive fitness. They offer several arguments to the effect that, in many cases, predictive and vernacular fitness simply cannot be related, and that in any event, the connection offered by the PIF is the wrong one.

The statisticalist interpretation goes further than this, on two fronts. First, vernacular fitness, they claim - due to its generality and, as we saw in the challenges above, the impossibility of specifying a universally valid formula connecting it to components of fitness - is not particularly useful in coming to understand biological 
populations. This is yet another place where the PIF has gone astray. It is rather the predictive fitness (and, in particular, the predictive fitness of traits and not of individual organisms) that is the appropriate target for biological study (Walsh, Lewens, and Ariew 2002; Walsh 2003, 2004, 2007).

Second, there is a further, and more important, reason that a singular conceptual picture of vernacular fitness fails. A particular fitness distribution can be the result of many different types of causes. We might, in one population, determine that some sexratio strategy is beneficial, and that in another population a particular parental care strategy is beneficial (Matthen and Ariew 2002, p. 67). In both cases, we are justified in saying that the outcomes at issue increase the fitness of the respective organisms. But to phrase this change in terms of a general notion of vernacular fitness is, the statisticalist interpretation argues, to miss the point. While both strategies may have "high" vernacular fitness - perhaps even both instantiate the same fitness distribution - the causes of this distribution are radically different, and the PIF fails, it is claimed, to recognize or sufficiently account for this fact.

Finally, one more target of the statisticalist line of argument brings us into contact with an aspect of the PIF that was only briefly mentioned above. In addition to the idea that fitness is derivable from the propensity to produce offspring, and that it should be measured by expected offspring number, the "traditional" PIF claimed that fitness plays a particular sort of causal role in natural selection. On this view, when we say that, on a standard view of natural selection, fitter organisms will outperform the less fit, we mean that (at least in part) this higher fitness is a cause of this higher performance. The fitness of individuals is causally responsible for their evolutionary success. 
The statisticalist interpretation also strongly rejects this claim. Rather than playing a causal role in the biological world, fitness - along with natural selection and genetic drift - are merely pragmatically useful ways to summarize events that take place in the biological world. There is nothing causally significant about these summaries, and it is only prudence and the good judgment of investigators that leads us to sometimes measure fitness in one way, sometimes in another. See also: DOI:

10.1002/9780470015902.a0005444.pub2

The claims of the statisticalist interpretation constitute a dramatic revision of our conceptual structure for fitness, selection, and drift, and have therefore been hotly contested in the philosophical literature - see, e.g., Matthen (2009), Matthen and Ariew (2009), Walsh (2010), and Lewens (2010) in support, and Reisman and Forber (2005), Brandon and Ramsey (2006), Millstein (2006), Abrams (2007), Shapiro and Sober (2007), Gildenhuys (2009), and Ramsey (forthcoming) in opposition.

\section{Responses in Defense of the PIF}

Several other authors, meanwhile, have offered ways in which to salvage the central insights of the PIF, attempting to sidestep the problems developed above. One modification of the PIF, originally proposed by Beatty and Finsen (1989) and elaborated by Brandon (1990), is intended to address the problem of reducing the PIF to a single numerical value. Perhaps it was a mistake, then, to think that one single way of making this reduction was suitable in all circumstances and that, as discussed in challenge 2 , the

arithmetic mean does not, in general, work as a mathematical model of the PIF. Brandon (1990, p. 20) modified the original expected-value formula for fitness, introducing a 
"correction factor" intended to compensate for the effects of influences like variance.

This correction factor takes the form of a function of the environment and the variance, added to the expected number of offspring, which Brandon termed $f\left(E, \sigma^{2}\right)$. This makes the PIF provide not a single numerical value for individual fitness, but a "schema" of possible equations, each of which needs to be filled out given the details of the particular population to be measured, the distribution of variation within it, and so forth. Such a defense could preserve the central insights of the PIF, at the cost of losing a mathematically unified definition of fitness (see also Abrams 2009).

A related approach, taken by Pence and Ramsey (forthcoming), is to simply develop a more complicated conceptual and mathematical formulation of the PIF that can manage to avoid the objections developed above. While the PIF understands organismic fitness as a propensity of individuals to have offspring, it says nothing about how that propensity should be analyzed conceptually. The model offered by Pence and Ramsey thus attempts to provide a more detailed vocabulary in which to describe this propensity, and with it a more intricate way in which to compute a numerical measure of fitness, drawn from adaptive dynamics. The Pence and Ramsey proposal is attempting to at once address challenges 2-4.

Implicit in the Pence and Ramsey approach is another way to defend the PIF, originally mentioned by Brandon (1990) and developed in more detail by Millstein (forthcoming). As we have stated several times, the PIF has multiple interrelated components. One of these, which we might call the "nonmathematical" portion of the PIF, is the claim that the fitness of an organism can be understood in terms of its propensity to produce offspring. Another, the "mathematical" portion of the PIF, is the 
claim that the best measure of this propensity is the expected number of offspring. Both the responses we have just seen have attempted to salvage this mathematical portion of the PIF. But is this really necessary? Millstein (forthcoming) argues that we might be better served if we can separate these two questions. Discarding this mathematical approach, and arguing for the propensity interpretation as an answer to the question of what fitness is, she claims, can evade many of these posed difficulties while leaving the question of the comparison of fitness distributions as a problem for mathematical biology. This addresses challenges $2-4$ by arguing that the PIF does not stand or fall based on the quality of the mathematical models with which it is associated.

Finally, concerning challenge 1, does the difficulty of measuring the quantity described by the PIF undercut it as an interpretation of fitness? One response is to point out that there are multiple possible roles that an interpretation of fitness can play. If an interpretation plays one role well (serving as a theoretical foundation for fitness, say) it does not follow that it should also be useful for another (such as studying evolution in natural populations with limited data). On this response, the PIF is offered not as a measurable model for fitness in experimental studies (Endler 1986), but rather as a way to ground theoretical considerations of natural selection in general-discussions of selection that are supposed to apply to every possible natural population, like those of Lewontin (1970) or Thoday (1953).

\section{Conclusions}

The debate over the best understanding of biological fitness has stood as a central problem in philosophical work on evolutionary theory for nearly twenty-five years, 
beginning with the rejection of the definition of fitness as actual contribution of offspring to the next generation. The "traditional" version of the propensity interpretation of fitness certainly now shows its age, and has accumulated several counterexamples that are quite probably fatal. Several plausible ways forward are apparent - from moderate revisions of the PIF or novel ways to argue for its central insights, to the complete rejection of the PIF and a view of fitness as a statistical predictor set by the interests of particular investigators. This debate, as well, has ramifications for the ways in which we see natural selection, genetic drift, and indeed the entire conceptual structure of evolutionary theory. See also: DOI: 10.1002/9780470015902.a0005104.pub2, DOI: 10.1038/npg.els.0001706, DOI: 10.1038/npg.els.0001750, DOI: 10.1038/npg.els.0003463, DOI:

10.1038/npg.els.0001698.

References [up to 50 items]

Abrams M (2007) How do natural selection and random drift interact? Philosophy of Science 74: 666-679.

Abrams M (2009) The unity of fitness. Philosophy of Science 76: 750-761.

Ahmed S, Hodgkin J (2000) MRT-2 checkpoint protein is required for germline immortality and telomere replication in C. elegans. Nature 403: 159-164. doi: $10.1038 / 35003120$.

Ariew A and Lewontin RC (2004) The confusions of fitness. British Journal for the Philosophy of Science 55: 347-363. doi: 10.1093/bjps/55.2.347.

Beatty JH, Finsen S (1989) Rethinking the propensity interpretation of fitness: A peek inside Pandora's box. In: Ruse M (ed) What the philosophy of biology is: Essays for David Hull. Kluwer Academic Publishers, Dordrecht, pp 18-30.

Bouchard F, Rosenberg A (2004) Fitness, probability and the principles of natural selection. British Journal for the Philosophy of Science 55: 693-712. doi: 10.1093/bjps/55.4.693. 
Brandon RN (1978) Adaptation and evolutionary theory. Studies in History and Philosophy of Science 9: 181-206. doi: 10.1016/0039-3681(78)90005-5.

Brandon RN (1990) Adaptation and environment. Princeton University Press, Princeton, NJ.

Brandon RN, Ramsey G (2006) What's wrong with the emergentist statistical interpretation of natural selection and random drift? In: Hull DL, Ruse M (eds) The Cambridge companion to the philosophy of biology. Cambridge University Press, Cambridge, pp 66-84.

Butler S (1879) Evolution old and new; or, the theories of Buffon, Dr. Erasmus Darwin, and Lamarck, as compared with that of Mr. Charles Darwin. Hardwick and Bogue, London.

Crow J, Kimura M (1956) Some genetic problems in natural populations. Proceedings of the third Berkeley symposium on mathematical statistics and probability 4: 1-22.

Darwin C (1859) On the origin of species. John Murray, London.

Endler JA (1986) Natural selection in the wild. Princeton University Press, Princeton, NJ.

Gildenhuys P (2009) An explication of the causal dimension of drift. British Journal for the Philosophy of Science 60: 521-555. doi: 10.1093/bjps/axp019.

Gillespie JH (1974) Natural selection for within-generation variance in offspring number. Genetics 76: 601-606.

Godfrey-Smith P (2009) Darwinian populations and natural selection. Oxford University Press, Oxford.

Hodge MJS (1987) Natural selection as a causal, empirical, and probabilistic theory. In: Krüger L, Gigerenzer G, Morgan MS (eds) The probabilistic revolution. The MIT Press, Cambridge, MA, pp 233-270.

Lewens T (2010) The natures of selection. British Journal for the Philosophy of Science 61: 313-333. doi: 10.1093/bjps/axp041.

Lewontin RC (1970) The units of selection. Annual Review of Ecology and Systematics 1: $1-18$.

Lewontin RC and Cohen D (1969) On population growth in a randomly varying environment. Proceedings of the National Academy of Sciences 62: 1056-1060.

Matthen M (2009) Drift and "statistically abstractive explanation". Philosophy of Science 76: 464-487. doi: 10.1086/648063. 
Matthen M, Ariew A (2002) Two ways of thinking about fitness and natural selection. Journal of Philosophy 99: 55-83.

Matthen M, Ariew A (2009) Selection and causation. Philosophy of Science 76: 201-224. doi: $10.1086 / 648102$.

Mayr E (1961) Cause and effect in biology. Science 134: 1501-1506.

Mills SK, Beatty JH (1979) The propensity interpretation of fitness. Philosophy of Science 46: 263-286.

Millstein RL (2006) Natural selection as a population-level causal process. British Journal for the Philosophy of Science 57: 627-653. doi: 10.1093/bjps/ax1025.

Millstein RL (forthcoming) Probability in biology: the case of fitness. In: Hájek A and Hitchcock C (eds) The Oxford Handbook of Probability and Philosophy. Oxford University Press, Oxford.

Pence $\mathrm{CH}$ and Ramsey $\mathrm{G}$ (forthcoming) A new foundation for the propensity interpretation of fitness. British Journal for the Philosophy of Science.

Pennock RT (1999) Tower of Babel: the evidence against the New Creationism. MIT Press: Cambridge, MA.

Pigliucci M and Kaplan JM (2006) Making sense of evolution: the conceptual foundations of evolutionary theory. University of Chicago Press, Chicago.

Popper K (1974) Darwinism as a metaphysical research programme. In: Schilpp PA (ed) The philosophy of Karl Popper. Open Court Press, New York and Chicago, pp 133143.

Ramsey G (forthcoming) Can fitness differences be a cause of evolution? Philosophy and Theory in Biology.

Reisman K, Forber P (2005) Manipulation and the causes of evolution. Philosophy of Science 72: 1113-1123.

Rosenberg A (1982) On the propensity definition of fitness. Philosophy of Science 49: 268-273.

Shapiro L and Sober E (2007) Epiphenomenalism - the do's and the don'ts. In: Wolters G, Machamer P (eds) Thinking about causes: from Greek philosophy to modern physics. University of Pittsburgh Press, Pittsburgh, PA, pp 235-264. 
Sober E (2001) The two faces of fitness. In: Singh RS (ed) Thinking about evolution: historical, philosophical, and political perspectives. The MIT Press, Cambridge, MA, pp 309-321.

Thoday JM (1953) Components of fitness. Symposia of the Society for Experimental Biology 7: 96-113.

Walsh DM (2003) Fit and diversity: explaining adaptive evolution. Philosophy of Science 70: $280-301$. doi: $10.1086 / 375468$.

Walsh DM (2004) Bookkeeping or metaphysics? The units of selection debate. Synthese 138: 337-361. doi: 10.1023/B:SYNT.0000016426.73707.92.

Walsh DM (2007) The pomp of superfluous causes: the interpretation of evolutionary theory. Philosophy of Science 74: 281-303. doi: 10.1086/520777.

Walsh DM (2010) Not a sure thing: fitness, probability, and causation. Philosophy of Science 77: 147-171. doi: 10.1086/651320.

Walsh DM, Lewens T, Ariew A (2002) The trials of life: natural selection and random drift. Philosophy of Science 69: 429-446. doi: 10.1086/342454.

\section{Further Reading}

Abrams M (2012) Measured, modeled, and causal conceptions of fitness. Frontiers in Genetics 3: 196. doi: 10.3389/fgene.2012.00196.

Beatty JH (1992) Fitness: theoretical contexts. In: Keller EF, Lloyd EA (eds) Keywords in evolutionary biology. Harvard University Press, Cambridge, MA and London, pp $115-119$.

De Jong G (1994) The fitness of fitness concepts and the description of natural selection. Quarterly Review of Biology 69: 3-29.

Henle K (1991) Some reflections on evolutionary theory, with a classification of fitness. Acta Biotheoretica 39: 91-106.

Ramsey G (2006) Block fitness. Studies in History and Philosophy of Biological and Biomedical Sciences 37:484-98. doi: 10.1016/j.shpsc.2006.06.009. 
Roff DA (2008) Defining fitness in evolutionary models. Journal of Genetics 87: 339348.

Sober E (2000) Philosophy of biology, 2nd ed. Westview Press, Boulder, CO.

Sober E (2010) Evolutionary theory and the reality of macro probabilities. In: Eells E, Fetzer J (eds) Probability in science. Springer, pp 133-162.

\section{Glossary}

arithmetic mean: a simple average; often not the most accurate way to predict the success of an organism or trait in future generations, especially in randomly varying environments or cases of high variance in outcomes

dispositional property: a property that describes how an object will act in certain kinds of circumstances (e.g., a soluble object dissolves if placed in an appropriate solvent); contrast with categorical properties, which describe how objects are (e.g., the property of massiveness)

ecological interpretation of fitness: an interpretation of fitness on which one organism is considered to be fitter than another if that organism successfully solves more design problems than its competitor (Bouchard and Rosenberg 2004)

geometric mean: the $n$-th root of the product of a series of $n$ numbers; often a better predictor of the success of an organism or trait in future generations in cases in which variance is high

propensity: a property which grounds how an object will behave probabilistically in certain circumstances (e.g., a coin's propensity to land heads-up when flipped)

propensity interpretation of fitness (PIF): an interpretation of fitness on which the fitness of an organism is grounded in its propensity to produce offspring (i.e., the probabilities that it has to produce $n$ offspring in the next generation)

statisticalist interpretation: an interpretation of fitness (and also natural selection and genetic drift) on which the fitness of an organism is merely a useful statistical measure of its future growth, not a description of a genuine causal influence

variance: a statistical measure of the "spread" of a set of outcomes; in cases where high variance is found in reproductive outcomes, the arithmetic mean is a poor predictor for long-term success 